\title{
Suppression of MKL1 promotes adipocytic differentiation and reduces the proliferation of myxoid liposarcoma cells
}

\author{
YOHEI KAMIKAWA ${ }^{*}$, KENTO YOKOTA ${ }^{*}$, KOSUKE OIKAWA, FUYUKI SATO and YASUTERU MURAGAKI \\ Department of Pathology, Wakayama Medical University, Wakayama 641-8509, Japan
}

Received January 31, 2020; Accepted September 23, 2020

DOI: $10.3892 / \mathrm{ol} .2020 .12232$

\begin{abstract}
Myxoid liposarcoma (MLS) is thought to occur due to defective adipocytic differentiation in mesenchymal stem cells. A promising strategy for MLS treatment is the prevention of sarcomagenesis by promoting the terminal differentiation of MLS cells into adipocytes. Previous studies have reported that the suppression of megakaryoblastic leukemia 1 (MKL1) expression induces adipocytic differentiation in preadipocyte cell lines. The present study aimed to investigate the effects of MKL1 suppression on MLS cells. In the present study, MKL1 knockdown was demonstrated to promote the adipocytic differentiation of an MLS-derived cell line, designated 1955/91, under adipogenic conditions. This suggests that therapeutic targeting of the MKL1-associated molecular pathway has potential as a promising method of MLS treatment. However, the induction of adipogenesis by MKL knockdown was incomplete, and Oil Red O staining indicated that intracellular lipid droplets were only sporadically generated. Conversely, MKL1 knockdown reduced the growth of the MLS cells. As adipocytic differentiation in vitro requires cellular confluence, the decreased growth rate of the MLS cells following MKL1 knockdown could be attributed to the incomplete induction of adipogenesis. Translocated in liposarcoma-CCAAT/enhancer-binding protein homologous protein (TLS-CHOP) is an MLS-specific oncoprotein that is thought to play key roles in sarcomagenesis and the suppression of adipocytic differentiation. However, the results of western blotting analyses suggest that TLS-CHOP has limited effects on MKL1 expression in MLS cells and that MKL1 knockdown hardly affects TLS-CHOP expression. Thus, it is postulated that the inhibitory effect of TLS-CHOP on adipogenesis is not associated with MKL1 expression. However, MKL1 and the
\end{abstract}

Correspondence to: Dr Kosuke Oikawa, Department of Pathology, Wakayama Medical University, 811-1 Kimiidera, Wakayama, Wakayama 641-8509, Japan

E-mail: k-oikawa@wakayama-med.ac.jp

*Contributed equally

Key words: myxoid liposarcoma, MKL1, adipocytic differentiation, differentiation therapy, Oil Red O staining molecular pathway involving MKL1 appear to be attractive targets for the differentiation therapy of MLS.

\section{Introduction}

Mesenchymal stem cells are pluripotent cells that possess the potential to differentiate into myocytic, osteocytic, chondrocytic and adipocytic cells (1-4). Several types of sarcoma are considered to be induced by differentiation defects in mesenchymal stem cells (5). Normal differentiation is accompanied by growth arrest, whereas sarcoma cells retain their proliferative capacity via the suppression of terminal differentiation (6). Thus, the induction of terminal differentiation appears to be an attractive therapeutic strategy for sarcomas. Differentiation therapies would be expected to suppress tumor proliferation and avoid the morbidity induced by current chemotherapies.

Liposarcoma is one of the most common sarcomas in adults (7). Myxoid liposarcoma (MLS), characterized by the chimeric oncoproteins translocated in liposarcoma (TLS)-CCAAT/enhancer-binding protein homologous protein (CHOP) in most cases or Ewing's sarcoma-CHOP in rare cases, is a major subtype of liposarcoma $(8,9)$. TLS is also known as fused in sarcoma, and CHOP has the alternative names DNA-damage-inducible transcript 3 and growth arrest- and DNA damage-inducible gene 153. Previous studies have shown that the antitumor compound trabectedin (also termed ET-743) promotes the adipocytic differentiation of MLS $(10,11)$. Furthermore, in our previous study, it was demonstrated that the knockdown of TLS-CHOP or its downstream molecule proteoglycan 4 promoted adipogenesis in an MLS-derived cell line under adipogenic conditions (12). However, the degree of adipocytic differentiation induced in these previous studies appeared to be insufficient to provide comprehensive recovery from MLS. Therefore, further studies are needed to establish an effective strategy for the differentiation therapy of MLS.

Megakaryoblastic leukemia 1 (MKL1), also known as myocardin-related transcription factor- $\mathrm{A}$, is a transcriptional coactivator affecting various biological mechanisms, including epithelial-mesenchymal transition (13), epidermal cell fate decisions (14), skeletal myogenic differentiation (15), circadian rhythm $(16)$ and plasticity of the nervous system $(17,18)$. Moreover, MKL1 is involved in the regulation of the expression of approximately one thousand genes (16). Previous studies have revealed that the knockdown of MKL1 in mouse preadipocyte cell lines drives adipocytic differentiation $(19,20)$. Thus, the 
knockdown of MKL1 in MLS cells is also expected to stimulate adipogenesis. Conversely, MKL1 was initially identified as part of the fusion protein RNA-binding motif protein-15 (RBM15)-MKL1 in acute megakaryoblastic leukemia $(21,22)$. Notably, RBM15-MKL1 is thought to play an important role in oncogenesis. Thus, the aberrant control of MKL1 expression may have oncogenic effects in other types of cells.

In the present study, the effects of MKL1 knockdown on the differentiation and growth of MLS cells were examined. The results may suggest possibilities for the development of a novel differentiation therapy targeting MKL1.

\section{Materials and methods}

Cell line. Human MLS-derived 1955/91 cells, as previously described (12), were maintained in Dulbecco's modified Eagle's medium (DMEM; D5796; Sigma-Aldrich; Merck KGaA) containing $10 \%$ fetal bovine serum (FBS; Biowest; https://www.biowest.net) at $37^{\circ} \mathrm{C}$ in a $5 \% \mathrm{CO}_{2}$ environment. The cells were provided by Professor Masahiko Kuroda (Tokyo Medical University).

Cell quantification was performed as previously described (23). For each experiment, cell numbers were calculated from four independent counts using a hemocytometer.

Small interfering RNA (siRNA) transfection and adipocytic differentiation assay. For siRNA transfection, cells were transfected with siRNA (20 nM final concentration) using Lipofectamine RNAiMAX Transfection Reagent and OPTI-MEM I Reduced Serum Medium (both Thermo Fisher Scientific, Inc.) as previously described (12). TLS-CHOP and negative control siRNAs were as previously described (24), and the target sequence of the MKL1 siRNA was 5'-CAT GGAGCTGGTGGAGAAGAA-3', as previously designed by Varney et al (25).

For the adipocytic differentiation assay, a MilliporeSigma Chemicon Adipogenesis Assay Kit (ECM950; Thermo Fisher Scientific, Inc.) and calcium (+)-pantothenate (FUJIFILM Wako Pure Chemical Corporation) were used. One-day preconfluent 1955/91 cells grown in growth medium (DMEM containing $10 \%$ FBS) were transfected with MKL1 siRNA or negative control siRNA. The next day, the cells reached confluence, and the medium was replaced with initiating medium (DMEM containing 10\% FBS, $100 \mu \mathrm{M}$ insulin, $250 \mu \mathrm{M}$ 3-isobutyl-1-methylxanthine, $1 \mu \mathrm{M}$ dexamethasone and $8.5 \mu \mathrm{M}$ calcium (+)-pantothenate). After 4 days, the medium was changed to maintenance medium (initiating medium without 3-isobutyl-1-methylxanthine) and the cells were incubated for a further 6 days. These culture media were replaced with fresh media every second day. The cells were then washed with phosphate-buffered saline (PBS) twice, incubated in Oil Red O Solution at room temperature for $15 \mathrm{~min}$, washed three times with wash solution, and observed using an inverted microscope (ECLIPSE TS100; Nikon Corporation) and a Microscope Camera Control Unit (DS-L3; Nikon Corporation).

Western blot analysis. Protein samples were prepared as previously described (26) and quantified using the Pierce ${ }^{\mathrm{TM}}$ BCA Protein Assay Kit (Thermo Fisher Scientific, Inc.). Samples containing equal amounts of protein $(10 \mu \mathrm{g} /$ lane $)$ were separated by SDS-PAGE (10\% gel) and transferred to Hybond ECL nitrocellulose membranes (Amersham; GE Healthcare). To confirm equal sample loading, the membranes were stained with Ponceau S solution (Sigma-Aldrich; Merck KGaA) for 1-2 min at room temperature. After blocking with 5\% skimmed milk powder (FUJIFILM Wako Pure Chemical Corporation) in PBS at room temperature for $1 \mathrm{~h}$, the membranes were probed with specific primary antibodies at room temperature overnight. The primary antibodies and dilutions used were as follows: Anti-TLS-CHOP antibody [1:2,500; clone 14; generated previously (27)]; MKL1 antibody (1:2,000; cat. no. A302-201A; Bethyl Laboratories, Inc.); peroxisome proliferator-activated receptor (PPAR $\gamma(81 \mathrm{~B} 8)$ rabbit monoclonal antibody $(1: 1,000$; cat.no.2443; Cell Signaling Technology,Inc.); cleaved caspase-3 (Asp175) antibody (1:1,000; cat. no. 9661; Cell Signaling Technology, Inc.); poly (ADP-ribose) polymerase (PARP) antibody (1:1,000; cat. no. 9542; Cell Signaling Technology, Inc.); CCAAT/enhancer-binding protein (C/EBP) $\beta$ antibody $(\mathrm{H}-7)$ (1:500; cat. no. sc-7962; Santa Cruz Biotechnology, Inc.); proliferating cell nuclear antigen (PCNA) antibody (FL-261) (1:5,000; cat. no. sc-7907; Santa Cruz Biotechnology, Inc.); minichromosome maintenance 2 (MCM2) antibody (E-8) (1:1,000; cat. no. sc-373702; Santa Cruz Biotechnology, Inc.); Ki67 antibody (1:1,000; cat. no. sc-23900; Santa Cruz Biotechnology, Inc.); monoclonal anti- $\alpha$-tubulin antibody (1:2,500; cat. no. T5168; Sigma-Aldrich; Merck KGaA); and anti-glyceraldehyde-3-phosphate dehydrogenase (GAPDH) antibody (1:20,000; cat. no. G8795; Sigma-Aldrich; Merck KGaA). The membranes were then washed with PBS three times, incubated in 5\% skimmed milk powder in PBS with goat anti-mouse IgG H\&L (ab205719; Abcam) or goat anti-rabbit IgG H\&L (ab205718; Abcam) at room temperature for $1 \mathrm{~h}$, and washed with PBS three times. The signals were visualized using ECL Prime Western Blotting Detection Reagent (Amersham; GE Healthcare) and detected with a Chemiluminescence CCD Imaging System (AE9300 Ez-Capture MG; ATTO Corporation).

Statistical analysis. Groups were compared using one-way analysis of variance followed by Turkey-Kramer's test. $\mathrm{P}<0.05$ was considered to indicate a statistically significant difference. Excel 2010 software (Microsoft Corporation), with the add-in software Statcel 3 (OMS Publishing Inc.; http://www. oms-publ.co.jp/), was used to perform the statistical analysis.

\section{Results}

MKL1 knockdown promotes adipocytic differentiation of MLS cells. Our previous study demonstrated that the human MLS-derived cell line 1955/91 can be induced to differentiate into adipocytes by the knockdown of certain MLS sarcomagenesis-associated proteins (12). Thus, 1955/91 cells were used in the present study to examine whether MKL1 knockdown stimulates the adipocytic differentiation of MLS cells. One day before the cells reached confluence, the 1955/91 cells cultured in growth medium were transfected with $M K L 1$-specific siRNA or negative control siRNA; the day of transfection was arbitrarily set as day 0 . On the next day (day 1), the cells appeared to be confluent as expected, and adipogenic 


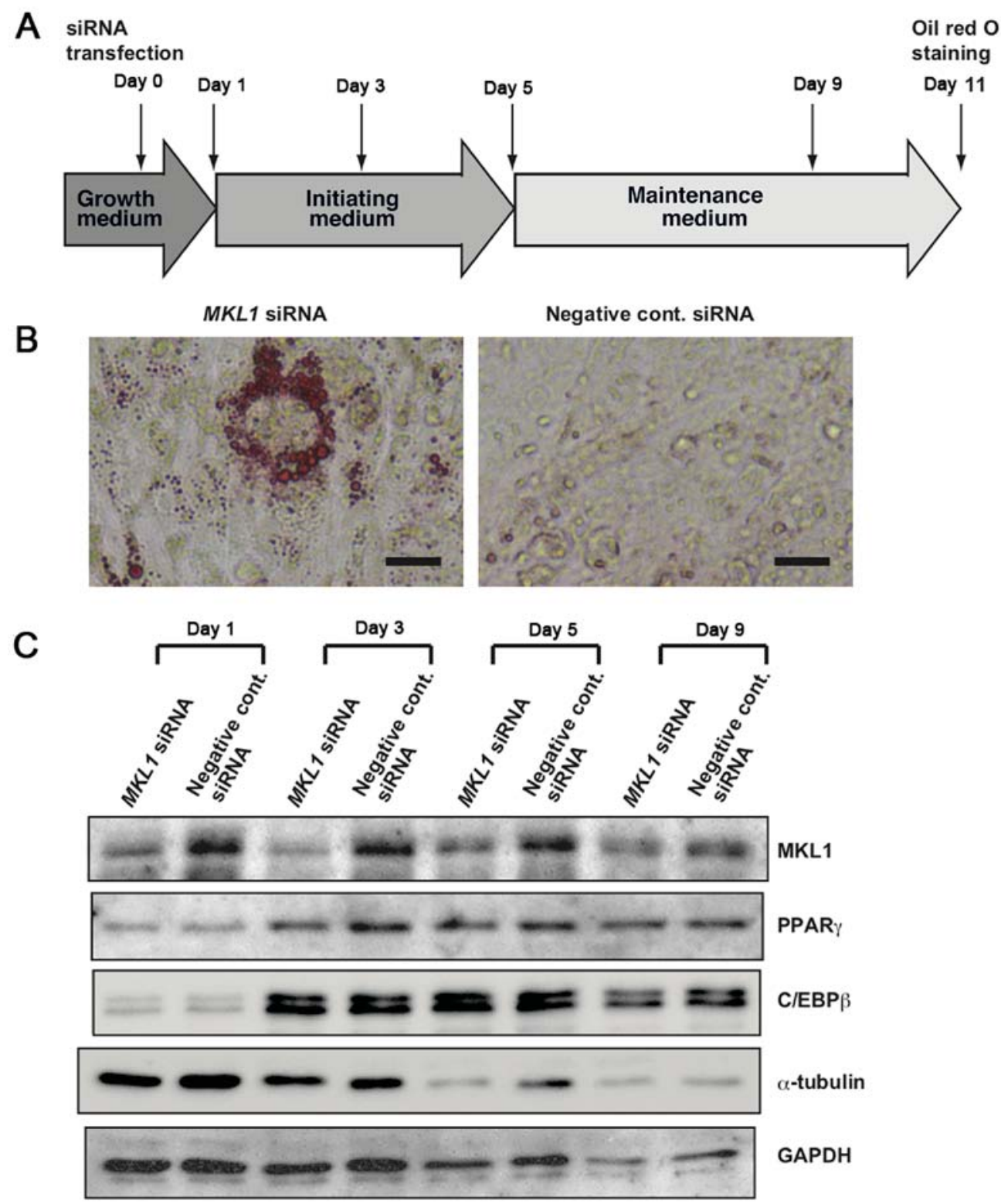

Figure 1. Adipocytic differentiation assay using myxoid liposarcoma-derived 1955/91 cells transfected with $M K L 1$ siRNA or negative control siRNA. (A) Protocol for the adipocytic differentiation assay. (B) Detection of lipid droplets in 1955/91 cells on day 11 of the adipocytic differentiation protocol by Oil Red O staining. Lipid droplets are visualized by red staining. Scale bars, $20 \mu \mathrm{m}$. (C) Western blot analysis of MKL1, PPAR $\gamma$ and C/EBP $\beta$ proteins on days 1, 3, 5 and 9 of the adipocytic differentiation assay. GAPDH and $\alpha$-tubulin are shown as loading controls. Results presented are representative of three independent experiments. MKL1, megakaryoblastic leukemia 1; siRNA, small interfering RNA; PPAR $\gamma$, peroxisome proliferator-activated receptor $\gamma$; C/EBP $\beta$, CCAAT/enhancer-binding protein $\beta$; GAPDH, glyceraldehyde-3-phosphate dehydrogenase.

stimulation of the cells was initiated by the addition of adipogenic agents in accordance with the adipocytic differentiation protocol illustrated in Fig. 1A. On day 11, the accumulation of lipid droplets in the cells was evaluated by Oil Red O staining, which stains neutral lipids. Oil Red O-positive cells were observed in the MKL1 siRNA-transfected cells, although the positive cells were sporadically distributed (Fig. 1B). However, most of the cells transfected with negative control siRNA did not exhibit any Oil Red O staining (Fig. 1B). Western blot analysis confirmed that $M K L 1$ siRNA markedly decreased MKL1 expression during adipocytic differentiation (Fig. 1C). Previous studies have shown that the expression of PPAR $\gamma$ promotes the adipogenesis of preadipocyte cells $(28,29)$, and that the knockdown of MKL1 increases PPAR $\gamma$ expression, as well as the transcriptional activity of PPAR $\gamma$ target genes $(19,20)$. Notably, PPAR $\gamma$ expression was observed to be induced by adipogenic agents during the adipocytic differentiation process in the present study (Fig. 1C). However, the induced levels of PPAR $\gamma$ in the MKL1-knockdown cells were lower, not higher, than those in the control cells on day 3 . Furthermore, $\mathrm{C} / \mathrm{EBP} \beta$ expression was examined in the present study (Fig. 1C). C/EBP $\beta$ plays multiple essential roles during adipogenesis (30). C/EBP $\beta$ expression was also induced by adipogenic agents, with no marked difference observed in C/EBP $\beta$ expression levels between the MKL1-knockdown cells and control cells. GAPDH and $\alpha$-tubulin expression levels were also examined as controls. Interestingly, a remarkable reduction in the $\alpha$-tubulin level was observed in the MKL1-knockdown cells on day 5 when compared with the control cells (Fig. 1C). 

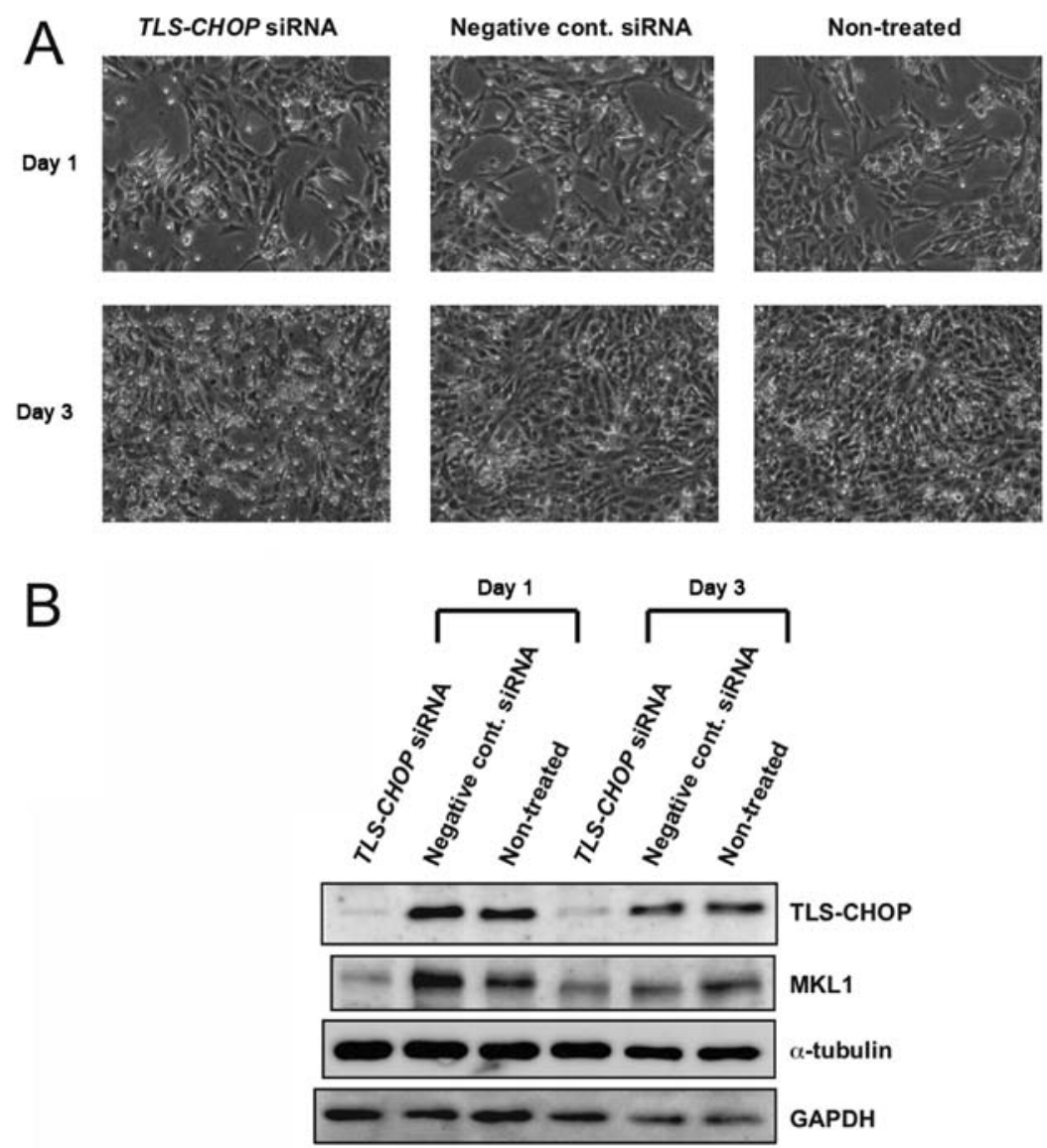

Figure 2. Effect of TLS-CHOP knockdown on MKL1 expression in myxoid liposarcoma-derived 1955/91 cells. (A) Representative phase-contrast images of 1955/91 cells on days 1 and 3 after siRNA transfection (magnification, x40). (B) Western blot analysis of TLS-CHOP and MKL1 proteins in 1955/91 cells on days 1 and 3 after siRNA transfection. GAPDH and $\alpha$-tubulin are shown as loading controls. Results shown are representative of three independent experiments. TLS-CHOP, translocated in liposarcoma-CCAAT/enhancer-binding protein homologous protein; MKL1, megakaryoblastic leukemia 1; siRNA, small interfering RNA; GAPDH, glyceraldehyde-3-phosphate dehydrogenase.

Effect of TLS-CHOP on MKL1 expression. Previous studies have reported that one of the oncogenic functions of the MLS-specific fusion oncoprotein, TLS-CHOP, is the inhibition of adipocytic differentiation $(31,32)$. This prompted an investigation of whether MKL1 expression is induced by TLS-CHOP. The MLS-derived 1955/91 cells at $\sim 20 \%$ confluence were transfected with $T L S-C H O P$ siRNA or negative control siRNA. As previously reported, the knockdown of TLS-CHOP by specific siRNA inhibited the growth of MLS cells (Fig. 2A) (24). The cells were harvested on days 1 and 3 post-transfection and protein samples prepared from the cells were examined by western blotting. Although MKL1 expression was inhibited by TLS-CHOP knockdown on day 1 after siRNA transfection, no notable reduction in MKL1 expression was observed in the TLS-CHOP-knockdown cells when compared with the control cells on day 3 after siRNA transfection (Fig. 2B).Thus, TLS-CHOP may have limited effects on MKL1 expression in MLS cells.

MKL1 knockdown reduces the proliferation of MLS cells. In the adipocytic differentiation assay illustrated schematically in Fig. 1, cells transfected with MKL1 siRNA and those transfected with negative control siRNA appeared to reach confluence on day 1 after transfection. However, it was suspected that the densities of these 'confluent' cell cultures moderately differed. Thus, the effect of MKL1 knockdown on the proliferation of MLS-derived 1955/91 cells was examined. As shown in Fig. 3A, MKL1 siRNA reduced the growth of MLS cells under normal growth conditions. Using western blot analysis, MKL1 knockdown in MKL1 siRNA-transfected cells was confirmed (Fig. 3B). On day 3 post siRNA transfection, TLS-CHOP expression appeared to be marginally decreased in the MKL1-knockdown cells compared with the control cells (Fig. 3B), suggesting that MKL1 knockdown failed to affect TLS-CHOP expression. Furthermore, MKL1 knockdown did not induce PPAR $\gamma$ and C/EBP $\beta$ expression (Fig. 3B).

Additionally, the expression levels of several cell proliferation markers, namely PCNA, MCM2 and Ki67, were explored (Fig.3B).PCNA levels were not reduced in the MKL1-knockdown cells compared with the control cells. However, MKL1 knockdown suppressed the expression of MCM2 and Ki67.

Furthermore, the expression of some apoptosis markers, namely cleaved caspase-3 and cleaved PARP, were investigated (Fig. 3B). Interestingly, the presence of cleaved caspase-3 and cleaved PARP signals was detected even in control cells, suggesting that apoptotic pathways may be innately induced to a certain extent in MLS-derived 1955/91 cells. Although notable differences in the levels of cleaved PARP between the MKL1-knockdown and control cells were not observed, cleaved caspase-3 levels were increased in the MKL1-knockdown cells 
A
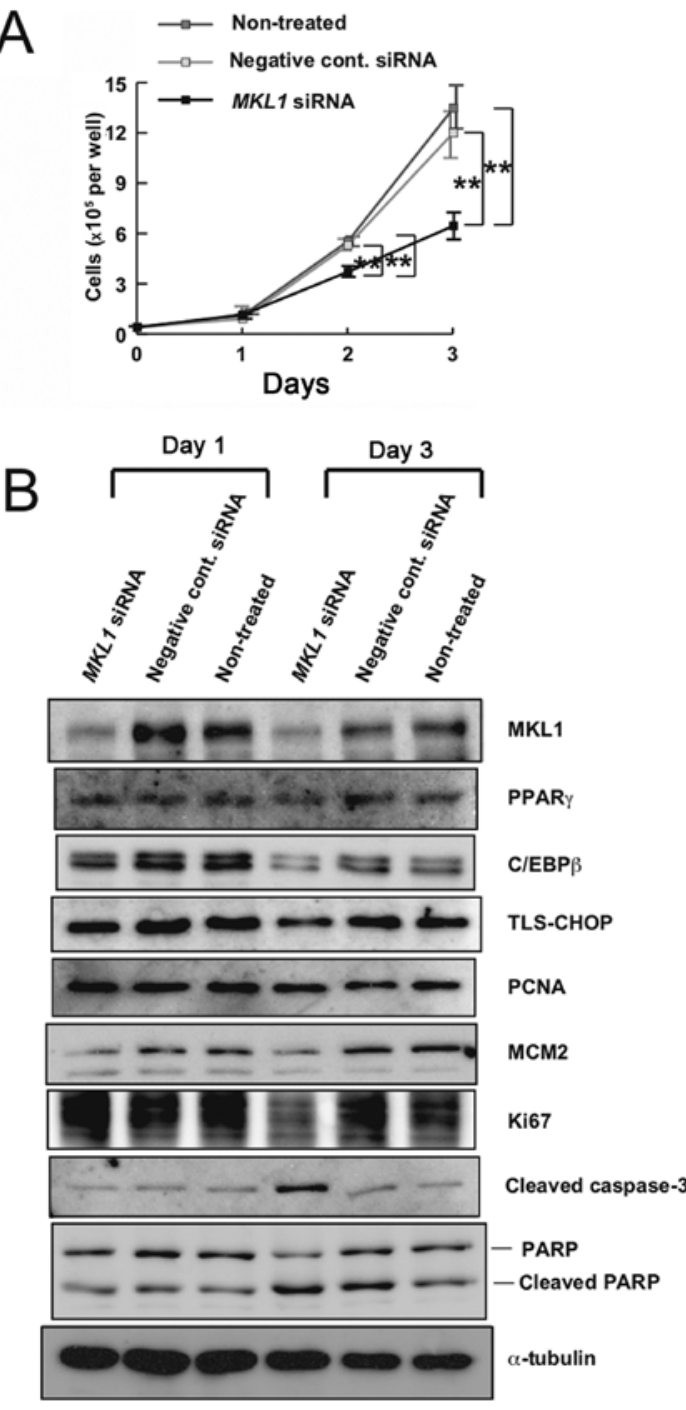

Figure 3. Effect of MKL1 knockdown on the growth of 1955/91 cells (A) Growth curves of 1955/91 cells. After siRNA transfection (day 0), the cells in 12-well culture plates were quantified at several time points. Bars represent standard deviation. ${ }^{* *} \mathrm{P}<0.01$. (B) Western blot analysis of MKL1, PPAR $\gamma, \mathrm{C} / \mathrm{EBP} \beta, \mathrm{TLS}-\mathrm{CHOP}, \mathrm{PCNA}, \mathrm{MCM} 2, \mathrm{Ki} 67$, cleaved caspase-3, PARP and cleaved PARP in 1955/91 cells on days 1 and 3 post siRNA transfection. $\alpha$-tubulin is shown as a loading control. Results shown are representative of three independent experiments. MKL1, megakaryoblastic leukemia 1 siRNA, small interfering RNA; PPAR $\gamma$, peroxisome proliferator-activated receptor $\gamma$; C/EBP $\beta$, CCAAT/enhancer-binding protein $\beta$; TLS-CHOP, translocated in liposarcoma-CCAAT/enhancer-binding protein homologous protein; PCNA, proliferating cell nuclear antigen; MCM2, minichromosome maintenance 2; PARP, poly (ADP-ribose) polymerase.

compared with the control cells on day 3 after siRNA transfection (Fig. 3B).

\section{Discussion}

The present study demonstrated that the knockdown of MKL1 promoted the adipocytic differentiation of MLS cells. This result suggests that MKL1 may be a promising target for differentiation therapy in MLS. However, adipogenesis occurred sporadically under the experimental conditions used in the present study. This could be attributed to MKL1 being insufficiently knocked down, and thus failing to induce the complete differentiation of MLS cells. Therefore, the selection of more effective siRNA target sequences in the $M K L 1$ gene may be crucial. Conversely, MKL1 knockdown was observed to reduce the proliferation of MLS cells under normal growth conditions. In vitro adipogenesis requires non-proliferating progenitors to be induced by confluent contact inhibition before the cells are exposed to adipogenic agents $(33,34)$. Hence, MKL1 knockdown may have opposing effects on the induction of adipocytic differentiation. Thus, although MKL1 is an attractive target molecule for the development of a differentiation therapy for MLS, further investigations into the molecular functions of MKL1 are required.

To elucidate the cause of the reduced proliferation of MKL1-knockdown MLS cells observed under normal growth conditions, the expression levels of the proliferation markers MCM2, Ki67 and PCNA were examined. It was observed that MCM2 and Ki67 expression levels were reduced in MKL1-knockdown cells. However, PCNA expression levels did not show a marked difference between the MKL1-knockdown and control cells. The family of MCM proteins, including MCM2, are key molecules for the regulation of DNA replication. MCM proteins are stably expressed throughout the cell cycle. Ki67 is expressed in all proliferating cells, and its expression level fluctuates during the cell cycle. However, the function of Ki67 during the cell cycle is unclear. PCNA is expressed in proliferating cells and its expression increases markedly during the $\mathrm{S}$ phase. PCNA has important roles in DNA replication, DNA repair and cell cycle control. Owing to its role in DNA repair, PCNA is a less specific proliferation marker. PCNA expression may be detected not only in actively proliferating cells but also in DNA-damaged cells (35). Thus, the reduced proliferation of MKL1-knockdown cells may be explained by the reduced expression of MCM2 and Ki67. Additionally, the observation that PCNA protein expression was not reduced in the MKL1-knockdown cells could imply the existence of the cells undergoing DNA repair. The expression levels of apoptotic markers, namely cleaved caspase-3 and cleaved PARP, were also examined in the present study. Activated PARP promotes DNA repair, whereas cleaved caspase- 3 cleaves and inactivates PARP and induces apoptosis (36). Three days after MKL1 siRNA transfection, it was observed that cleaved caspase-3 levels were increased in MKL1-knockdown cells. This suggests that MKL1 knockdown may promote apoptotic pathways in MLS cells. However, both cleaved caspase- 3 and cleaved PARP were detected in all the cells examined, suggesting that apoptotic pathways may be activated to a certain extent in the cells. In MLS cells, the TLS-CHOP fusion oncoprotein comprises the N-terminal half of TLS fused to the full-length CHOP protein (24). CHOP is normally expressed at extremely low levels but is strongly induced by endoplasmic reticulum stress, and it cleaves and activates caspase-3 (37). Thus, the typically expressed TLS-CHOP may consistently activate caspase-3 to a certain degree in the MLS-derived cell line. Nevertheless, DNA damage and the promotion of apoptotic pathways appear to be involved in the reduced proliferation of MKL1-knockdown MLS cells. However, further studies are required to determine the relevant details.

In a previous study, Nobusue et al (19) demonstrated that the loss of MKL1 induces PPAR $\gamma$ expression sufficiently to drive the adipocytic differentiation of mouse preadipocyte cells. However, although MKL1 knockdown was required in addition to treatment with adipogenic agents to promote the adipogenesis 
of MLS cells under the experimental conditions of the present study, no further induction of PPAR $\gamma$ expression was observed in MKL1-knockdown cells compared with control cells at each time point during the adipogenesis induction process. These results suggest that MKL1 knockdown does not further increase the induction of PPAR $\gamma$ expression by adipogenic agents, at least in MLS cells. Adipocytic differentiation is regulated by a molecular cascade involving numerous transcription factors. $\mathrm{C} / \mathrm{EBP} \beta$ plays a central role in the cascade and induces the expression of two dominant transcription factors, $\mathrm{C} / \mathrm{EBP} \alpha$ and PPAR $\gamma$, for terminal adipocytic differentiation (30). In the present study, $\mathrm{C} / \mathrm{EBP} \beta$ expression was induced only by adipogenic agents, and MKL1 knockdown failed to demonstrate an additional inductive effect on $\mathrm{C} / \mathrm{EBP} \beta$ during the adipocytic differentiation process. Thus, we hypothesize that MKL1 knockdown may stimulate another novel and critical mechanism associated with adipocytic differentiation in MLS cells. In this case, MKL1 would become increasingly important for the promotion and inhibition of adipogenesis in MLS cells.

In the adipocytic differentiation assay, a marked reduction in the $\alpha$-tubulin level in MKL1-knockdown cells was observed on day 5 when compared with that in control cells. The biosynthetic rate for cytoskeletal proteins, including $\alpha$-tubulin, has been reported to be greatly reduced during adipocytic differentiation(38). Thus, the reduced level of $\alpha$-tubulinin MKL1-knockdown cells on day 5 may suggest the progression of adipocytic differentiation. Conversely, it was observed that $\alpha$-tubulin expression was reduced even in the negative control cells on day 9. The biosynthetic alteration of cytoskeletal proteins occurs at a very early stage during adipocytic differentiation (38), and the induction of PPAR $\gamma$ and C/EBP $\beta$ in both MKL1-knockdown cells and control cells was observed. Thus, we consider that the process of adipocytic differentiation also progressed to a certain extent in control cells. Furthermore, the expression level of GAPDH was reduced in both MKL1-knockdown and control cells on day 9. Previous studies have shown that the expression of GAPDH increases during adipocytic differentiation $(39,40)$. By contrast, the adipogenesis inhibitor berberine decreases GAPDH expression during adipocytic differentiation (40). Thus, some factors that inhibit adipogenesis in MLS cells may decrease GAPDH expression. Several previous studies have suggested suitable reference genes for quantitative real-time polymerase chain reaction during adipocytic differentiation (39-41). However, to the best of our knowledge, appropriate loading control proteins for western blot analysis during adipocytic differentiation have not yet been identified.

The MLS-specific fusion oncoprotein TLS-CHOP is considered an abnormal transcription factor associated with sarcomagenesis, tumor maintenance and the inhibition of adipogenesis $(31,32,42-44)$. Thus, we hypothesized that TLS-CHOP would induce MKL1 expression and consequently suppress adipocytic differentiation in MLS cells. However, the western blotting results suggest that TLS-CHOP has limited effects on MKL1 expression in MLS cells, at least, under the experimental conditions used in the present study. Additionally, MKL1 knockdown exhibited almost no effect on TLS-CHOP expression. Thus, the inhibition of adipogenesis by TLS-CHOP appears to have little impact on MKL1 expression. By contrast, a previous study reported that the downregulation of TLS-CHOP expression does not increase PPAR $\gamma$ expression, but PPAR $\gamma$ agonists enhance adipocytic differentiation via the downregulation of TLS-CHOP in endogenous mesenchymal stem cells in a mouse model of MLS (11). Based on these results, TLS-CHOP and MKL1 may inhibit adipogenesis by different mechanisms. Furthermore, TLS-CHOP may repress some downstream genes of PPAR $\gamma$. However, the results of the present study suggest that the mechanisms by which TLS-CHOP and MKL1 inhibit the adipocytic differentiation of MLS are complex.

\section{Acknowledgements}

The authors of the present study would like to thank Professor Masahiko Kuroda (Tokyo Medical University) for providing the MLS-derived 1955/91 cells.

\section{Funding}

The present study was supported by a Grant-in-Aid for Scientific Research (C) from the Japan Society for the Promotion of Science (grant no. 17K08768).

\section{Availability of data and materials}

The datasets shown and/or analyzed in the present study are available from the corresponding author upon reasonable request.

\section{Authors' contributions}

YK, KY, KO and YM designed the study. YK, KY and KO performed the experiments. YK, KY, KO and FS analyzed the data. KO and YM drafted the initial manuscript. All authors have read and approved the final manuscript.

\section{Ethics approval and consent to participate}

Not applicable.

\section{Patient consent for publication}

Not applicable.

\section{Competing interests}

The authors declare that they have no competing interests.

\section{References}

1. He BC, Chen L, Zuo GW, Zhang W, Bi Y, Huang J, Wang Y, Jiang W, Luo Q, Shi Q, et al: Synergistic antitumor effect of the activated PPARgamma and retinoid receptors on human osteosarcoma. Clin Cancer Res 16: 2235-2245, 2010.

2. Aubin JE: Regulation of osteoblast formation and function. Rev Endocr Metab Disord 2: 81-94, 2001.

3. Luu HH, Song WX, Luo X, Manning D, Luo J, Deng ZL, Sharff KA, Montag AG, Haydon RC and He TC: Distinct roles of bone morphogenetic proteins in osteogenic differentiation of mesenchymal stem cells. J Orthop Res 25: 665-677, 2007.

4. Deng ZL, Sharff KA, Tang N, Song WX, Luo J, Luo X, Chen J, Bennett E, Reid R, Manning D, et al: Regulation of osteogenic differentiation during skeletal development. Front Biosci 13: 2001-2021, 2008.

5. Reya T, Morrison SJ, Clarke MF and Weissman IL: Stem cells, cancer, and cancer stem cells. Nature 414: 105-111, 2001. 
6. Luther G, Rames R, Wagner ER, Zhu G, Luo Q, Bi Y, Kim SH, Gao JL, Huang E, Yang K, et al: Molecular basis of differentiation therapy for soft tissue sarcomas. Trends Cancer Res 6: $69-90,2010$

7. Fritchie KJ, Goldblum JR, Tubbs RR, Sun Y, Carver P Billings SD and Rubin BP: The expanded histologic spectrum of myxoid liposarcoma with an emphasis on newly described patterns: Implications for diagnosis on small biopsy specimens. Am J Clin Pathol 137: 229-239, 2012.

8. Dal Cin P, Sciot R, Panagopoulos I, Aman P, Samson I, Mandahl N, Mitelman F, Van den Berghe H and Fletcher CD Additional evidence of a variant translocation $\mathrm{t}(12 ; 22)$ with EWS-CHOP fusion in myxoid liposarcoma: Clinicopathologic features. J Pathol 182: 437-441, 1997.

9. Loubignac F, Bourtoul C and Chapel F: Myxoid liposarcoma: A rare soft tissue tumor with a misleading benign appearance. World J Surg Oncol 7: 42, 2009.

10. Forni C, Minuzzo M, Virdis E, Tamborini E, Simone M, Tavecchio M, Erba E, Grosso F, Gronchi A, Aman P, et al: Trabectedin (ET-743) promotes differentiation in myxoid liposarcoma tumors. Mol Cancer Ther 8: 449-457, 2009.

11. Charytonowicz E, Terry M, Coakley K, Telis L, Remotti F, Cordon-Cardo C, Taub RN and Matushansky I: PPAR $\gamma$ agonists enhance ET-743-induced adipogenic differentiation in a transgenic mouse model of myxoid round cell liposarcoma. J Clin Invest 122: 886-898, 2012.

12. Oikawa K, Mizusaki A, Takanashi M, Ozaki T, Sato F, Kuroda M and Muragaki Y: PRG4 expression in myxoid liposarcoma maintains tumor cell growth through suppression of an antitumor cytokine IL-24. Biochem Biophys Res Commun 485: 209-214, 2017.

13. Gomez EW, Chen QK, Gjorevski N and Nelson CM: Tissue geometry patterns epithelial-mesenchymal transition via intercellular mechanotransduction. J Cell Biochem 110: 44-51, 2010.

14. Connelly JT, Gautrot JE, Trappmann B, Tan DW, Donati G, Huck WT and Watt FM: Actin and serum response factor transduce physical cues from the microenvironment to regulate epidermal stem cell fate decisions. Nat Cell Biol 12: 711-718, 2010

15. Selvaraj A and Prywes R: Megakaryoblastic leukemia-1/2, a transcriptional co-activator of serum response factor, is required for skeletal myogenic differentiation. J Biol Chem 278: 41977-41987, 2003.

16. Esnault C, Stewart A, Gualdrini F, East P, Horswell S, Matthews N and Treisman R: Rho-actin signaling to the MRTF coactivators dominates the immediate transcriptional response to serum in fibroblasts. Genes Dev 28: 943-958, 2014

17. Kalita K, Kharebava G, Zheng JJ and Hetman M: Role of megakaryoblastic acute leukemia-1 in ERK1/2-dependent stimulation of serum response factor-driven transcription by BDNF or increased synaptic activity. J Neurosci 26: 10020-10032, 2006.

18. Kalita K, Kuzniewska B and Kaczmarek L: MKLs: Co-factors of serum response factor (SRF) in neuronal responses. Int J Biochem Cell Biol 44: 1444-1447, 2012.

19. Nobusue H, Onishi N, Shimizu T, Sugihara E, Oki Y, Sumikawa Y, Chiyoda T, Akashi K, Saya H and Kano K: Regulation of MKL1 via actin cytoskeleton dynamics drives adipocyte differentiation. Nat commun 5: 3368, 2014

20. Rosenwald M, Efthymiou V, Opitz L and Wolfrum C: SRF and MKL1 independently inhibit brown adipogenesis. PLoS One 12 e0170643, 2017.

21. Ma Z, Morris SW, Valentine V, Li M, Herbrick JA, Cui X, Bouman D, Li Y, Mehta PK, Nizetic D, et al: Fusion of two novel genes, RBM15 and MKL1, in the $t(1 ; 22)(\mathrm{p} 13 ; \mathrm{q} 13)$ of acute megakaryoblastic leukemia. Nat Genet 28: 220-221, 2001.

22. Mercher T, Coniat MB, Monni R, Mauchauffe M, Nguyen Khac F, Gressin L, Mugneret F, Leblanc T, Dastugue N, Berger R and Bernard OA: Involvement of a human gene related to the drosophila spen gene in the recurrent $t(1 ; 22)$ translocation of acute megakaryocytic leukemia. Proc Natl Acad Sci USA 98: $5776-5779,2001$

23. Oikawa K, Ohbayashi T, Kiyono T, Nishi H, Isaka K, Umezawa A, Kuroda M and Mukai K: Expression of a novel human gene, human wings apart-like (hWAPL), is associated with cervical carcinogenesis and tumor progression. Cancer Res 64: 3545-3549, 2004.

24. Oikawa K, Tanaka M, Itoh S, Takanashi M, Ozaki T, Muragaki Y and Kuroda M: A novel oncogenic pathway by TLS-CHOP involving repression of MDA-7/IL-24 expression. Br J Cancer 106: 1976-1979, 2012.
25. Varney SD, Betts CB, Zheng R, Wu L, Hinz B, Zhou J and Van De Water L: Hic-5 is required for myofibroblast differentiation by regulating mechanically dependent MRTF-A nuclear accumulation. J Cell Sci 129: 774-787, 2016.

26. Oikawa K, Ohbayashi T, Mimura J, Fujii-Kuriyama Y, Teshima S, Rokutan K, Mukai K and Kuroda M: Dioxin stimulates synthesis and secretion of IgE-dependent histamine-releasing factor. Biochem Biophys Res Commun 290: 984-987, 2002.

27. Oikawa K,Ishida T,Imamura T, YoshidaK, Takanashi M,HattoriH, Ishikawa A, Fujita K, Yamamoto K, Matsubayashi J, et al: Generation of the novel monoclonal antibody against TLS/EWS-CHOP chimeric oncoproteins that is applicable to one of the most sensitive assays for myxoid and round cell liposarcomas. Am J Surg Pathol 30: 351-356, 2006.

28. Tontonoz P, Hu E, Graves RA, Budavari AI and Spiegelman BM: mPPAR gamma 2: tissue-specific regulator of an adipocyte enhancer. Genes Dev 8: 1224-1234, 1994.

29. Chawla A, Schwarz EJ, Dimaculangan DD and Lazar MA Peroxisome proliferator-activated receptor (PPAR) gamma: Adipose-predominant expression and induction early in adipocyte differentiation. Endocrinology 135: 798-800, 1994.

30. Guo L, Li X, and Tang QQ: Transcriptional regulation of adipocyte differentiation: A central role for CCAAT/enhancer-binding protein (C/EBP) $\beta$. J Biol Chem 290: 755-761, 2015.

31. Batchvarova N, Wang XZ and Ron D: Inhibition of adipogenesis by the stressinduced protein CHOP (Gadd153). EMBO J 14: 4654-4661, 1995.

32. Kuroda M, Ishida T, Takanashi M, Satoh M, Machinami R and Watanabe T: Oncogenic transformation and inhibition of adipocytic conversion of preadipocytes by TLS/FUS-CHOP type II chimeric protein. Am J Pathol 151: 735-744, 1997.

33. Rosen ED and Spiegelman BM: Molecular regulation of adipogenesis. Annu Rev Cell Dev Biol 16: 145-171, 2000.

34. Gregoire FM, Smas CM and Sul HS: Understanding adipocyte differentiation. Physiol Rev 78: 783-809, 1998.

35. Juríková M, Danihel L, Polák S and Varga I: Ki67, PCNA, and MCM proteins: Markers of proliferation in the diagnosis of breast cancer. Acta Histochem 118: 544-552, 2016.

36. Malhotra U, Zaidi AH, Kosovec JE, Kasi PM, Komatsu Y, Rotoloni CL, Davison JM, Irvin CR, Hoppo T, Nason KS, et al: Prognostic value and targeted inhibition of survivin expression in esophageal adenocarcinoma and cancer-adjacent squamous epithelium. PLoS One 8: e78343, 2013.

37. Oyadomari S and Mori M: Roles of CHOP/GADD153 in endoplasmic reticulum stress. Cell Death Differ 11: 381-389, 2004

38. Spiegelman BM and Farmer SR: Decreases in tubulin and actin gene expression prior to morphological differentiation of 3T3 adipocytes. Cell 29: 53-60, 1982.

39. Arsenijevic T, Gre'goire F, Delforge V, Delporte C and Perret J: Murine 3T3-L1 adipocyte cell differentiation model: Validated reference genes for qPCR gene expression analysis. PLoS One 7: e37517, 2012 .

40. Zhang J, Tang H, Zhang Y, Deng R, Shao L, Liu Y, Li F, Wang X and Zhou L: Identification of suitable reference genes for quantitative RT-PCR during 3T3-L1 adipocyte differentiation. Int J Mol Med 33: 1209-1218, 2014.

41. Gentile AM, Lhamyani S, Coín-Aragüez L, Oliva-Olivera W, Zayed H, Vega-Rioja A, Monteseirin J, Romero-Zerbo SY, Tinahones FJ, Bermúdez-Silva FJ and Bekay RE: RPL13A and EEF1A1 are suitable reference genes for qPCR during adipocyte differentiation of vascular stromal cells from patients with different BMI and HOMA-IR. PLoS One 11: e0157002, 2016 .

42. Kuroda M, Wang X, Sok J, Yin Y, Chung P, Giannotti JW, Jacobs KA, Fitz LJ, Murtha-Riel P, Turner KJ and Ron D: Induction of a secreted protein by the myxoid liposarcoma oncogene. Proc Natl Acad Sci USA 96: 5025-5030, 1999.

43. Perez-Mancera PA, Bermejo-Rodriguez C, Sanchez-Martin M, Abollo-Jimenez F, Pintado B and Sanchez-Garcia I: FUS-DDIT3 prevents the development of adipocytic precursors in liposarcoma by repressing PPARgamma and C/EBPalpha and activating eIF4E. PLoS One 3: e2569, 2008.

44. Andersson MK, Goransson M, Olofsson A, Andersson C and Aman P: Nuclear expression of FLT1 and its ligand PGF in FUS-DDIT3 carrying myxoid liposarcomas suggests the existence of an intracrine signaling loop. BMC Cancer 10: 249, 2010 . 